Annuaire du Collège de France 2017-2018

\title{
Innovation technologique Liliane Bettencourt
}

\section{Thomas Ebbesen}

\section{OpenEdition}

Journals

Édition électronique

URL : https://journals.openedition.org/annuaire-cdf/16014

DOI : 10.4000/annuaire-cdf.16014

ISBN : 978-2-7226-0572-5

ISSN : 2109-9227

Éditeur

Collège de France

Édition imprimée

Date de publication : 30 décembre 2020

Pagination : 605-611

ISBN : 978-2-7226-0516-9

ISSN : 0069-5580

Référence électronique

Thomas Ebbesen, «Innovation technologique Liliane Bettencourt », L'annuaire du Collège de France [En ligne], 118 | 2020, mis en ligne le 01 avril 2021, consulté le 22 août 2022. URL : http://

journals.openedition.org/annuaire-cdf/16014 ; DOI : https://doi.org/10.4000/annuaire-cdf.16014 


\title{
INNOVATION TECHNOLOGIQUE LILIANE BETTENCOURT*
}

\author{
Thomas EBBESEN
}

Professeur de chimie physique et directeur de l'Institut d'études avancées de l'université de Strasbourg, professeur invité au Collège de France

La leçon inaugurale "L'alchimie du vide. Interactions lumière-matière en chimie physique » est disponible, en audio et vidéo, sur le site internet du collège de France (https://www.college-de-france.fr/site/thomas-ebbesen/inaugural-lecture2017-2018.htm) et a été publiée sous forme imprimée (Collège de France/Fayard, coll. " Leçons inaugurales ", 2019).

\section{ENSEIGNEMENT}

\section{COURS - LES INTERACTIONS LUMIÈRE-MATIÈRE EN CHIMIE PHYSIQUE}

\section{Leçon inaugurale - L'alchimie du vide. Interactions lumière-matière en chimie physique}

La leçon inaugurale du 2 mai 2018 et les cours qui ont suivi ont été centrés sur les interactions entre la lumière et la matière et sur leurs manifestations en chimie et dans les milieux moléculaires. Les interactions lumière-matière sont fondamentales pour l'existence de la vie et de la matière, telles que nous les connaissons, et elles sont au cœur de nos échanges culturels. Elles sont impliquées dans notre perception de notre environnement, dans l'échange d'information, le web, et dans les outils, de la chirurgie à la fabrication de voiture. Elles jouent ainsi un rôle prépondérant dans notre société moderne. En même temps, étant donné leur omniprésence, il est facile d'oublier leur importance.

Les plantes photosynthétiques, en particulier la chlorophylle, absorbent la lumière et par ce processus elles approvisionnent notre biosphère en énergie. La

\footnotetext{
* Chaire créée avec le soutien de la fondation Bettencourt Schueller.
} 
photosynthèse transforme l'énergie lumineuse du soleil en énergie chimique. La vie requiert constamment de l'énergie car un système vivant est hors équilibre. Un seul photon contient une énergie minuscule à l'échelle macroscopique, mais à l'échelle d'une molécule, la quantité d'énergie est énorme et elle modifie complètement sa chimie et la rend très réactive. Dans le cas de la photosynthèse, le flux de photons induit un transfert d'électrons entre les molécules, aboutissant au stockage d'une partie de l'énergie dans des molécules riches en énergie.

Les plantes n'absorbent qu'une fraction du spectre solaire, qui est lui-même très réduit à la surface de la Terre par rapport au spectre émis. Quand nous parlons de lumière, nous désignons typiquement des ondes électromagnétiques dans une gamme de longueurs d'onde très étroite - entre 400 et 700 nanomètres $(\mathrm{nm})$ - que nous sommes capables de détecter avec nos yeux et qui ne constitue qu'une infime partie du spectre électromagnétique possible. Au-dessus de $700 \mathrm{~nm}$, l'infrarouge solaire contribue à chauffer la Terre. En dessous de $400 \mathrm{~nm}$, la lumière solaire ultraviolette (UV) peut être nuisible ou bénéfique.

Par exemple, la couche d'ozone $\left(\mathrm{O}_{3}\right)$ et l'oxygène que nous respirons $\left(\mathrm{O}_{2}\right)$ nous protègent de la lumière solaire ultraviolette appelée UV-C (200 à $280 \mathrm{~nm})$ par un jeu de réactions photochimiques impliquant $\mathrm{O}_{2}$ et $\mathrm{O}_{3}$ qui absorbent l'UV-C. Par contre l'UV-B (280 à $315 \mathrm{~nm}$ ), un peu moins énergétique, est absorbé par une molécule analogue au cholestérol dans notre peau pour donner la vitamine D3, une photochimie essentielle à la vie. Depuis l'Antiquité, il existe des thérapies photochimiques pour traiter des maladies de la peau comme le psoriasis, l'eczéma et le vitiligo. Une version moderne est le traitement appelé PUVA - psoralène + UV-A (315-400 nm) où la molécule ingérée, le psoralène, s'insère entre les brins de l'ADN. Sous l'action photochimique de l'UV-A, un pontage chimique entre les deux brins se forme, qui bloque le fonctionnement normal de l'ADN, par exemple sa lecture par des enzymes. Il s'en suit l'apoptose de la cellule.

Ce qui peut paraître plus surprenant pour les non-physiciens est que les interactions entre la lumière et la matière interviennent même dans l'obscurité la plus totale. De manière plus générale, elles entrent en jeu dans les forces intermoléculaires, dans un liquide ou dans un tissu biologique, et ont donc un rôle vital. On ne peut pas vraiment comprendre ces types d'interaction sans mieux comprendre les propriétés du vide.

La nature du vide a été le sujet de débats depuis l'Antiquité. L'émergence de la physique quantique, puis de l'électrodynamique quantique avec les travaux de Paul Dirac dans les années 1920-1930 va de nouveau bouleverser notre compréhension du vide. Sans entrer dans les détails de cette physique, on réalise petit à petit que le vide matériel n'est pas vraiment «vide », mais plein de fluctuations électromagnétiques et de particules virtuelles, c'est-à-dire disparaissant aussitôt formées, ne laissant comme trace qu'une ombre de leur existence en perturbant les propriétés de la matière. Les propriétés du vide jouent ainsi un rôle déterminant dans beaucoup de processus naturels, au point que l'on ne peut pas imaginer l'existence de la matière telle qu'on la connaît sans les états du vide. Mais peut-on faire plus ? Par exemple jouer avec les états du vide pour modifier des propriétés de la matière ou la réactivité chimique ? Pour cela, il suffit de placer des molécules dans une cavité optique qui est résonante avec une transition de ces molécules. Dans les bonnes conditions, la résonance optique et la résonance moléculaire vont se coupler fortement pour former des états hybrides lumière-matière et ceci même dans l'obscurité la plus totale grâce aux fluctuations quantiques. Par ce moyen, nous avons pu démontrer pour la première fois la modification d'une réaction chimique sous couplage fort avec le 
champ électromagnétique du vide. Depuis cette preuve de principe, diverses réactions ont été étudiées sous couplage fort montrant qu'on peut favoriser un produit par rapport à une autre, que le mécanisme réactionnel peut être modifié et que les réactions peuvent être accélérées, c'est à dire une vraie catalyse induite par l'interaction physique avec la cavité.

Le couplage fort de matériaux à l'état solide ouvre aussi de nouvelles possibilités puisque cela peut aussi conduire à des modifications de propriétés (par exemple, la non-linéarité optique, la fonction de travail, le transport) ou encore faire émerger de nouvelles fonctionnalités (par exemple le transfert d'énergie par intrication quantique).

L'ingénierie de la matière par le contrôle des interactions avec l'environnement électromagnétique est relativement facile à mettre en œuvre, même si les fondements associés de l'électrodynamique quantique peuvent être surprenants et difficiles à conceptualiser. Dans le contexte de cette leçon inaugurale, quelques exemples seulement ont pu être donnés, et de manière sommaire, visant à donner des preuves de principe du potentiel que peut avoir le couplage fort lumière-matière. L'hybridation lumière-matière est un outil unique pour modifier les propriétés microscopiques et macroscopiques des matériaux moléculaires. Alors que les interactions lumière-matière ont été largement étudiées en physique fondamentale, il est évident que le couplage fort ouvre des perspectives très vastes, qui restent à explorer, dans le domaine des sciences des matériaux et des sciences moléculaires.

\section{Cours 1 - Photophysique moléculaire}

L'historique de la photochimie et photophysique moléculaire est intimement liée à l'utilisation de la photothérapie en médecine depuis l'Antiquité, l'observation de la luminescence au XVII ${ }^{\mathrm{e}}$ siècle ainsi que l'invention de la photographie au début du $\mathrm{XIX}^{\mathrm{e}}$ siècle. Les techniques de spectroscopies résolues dans le temps vont ensuite avoir un impact considérable sur la compréhension des processus photophysiques et photochimiques et de là sur la compréhension de la nature de la structure électronique des molécules et de la dynamique des états moléculaires dans la seconde moitié du $\mathrm{XX}^{\mathrm{e}}$ siècle. Ronald Norrish et George Porter (prix Nobel de chimie en 1967) vont développer des appareils avec des flashs photographiques donnant une résolution temporelle de la milliseconde à la microseconde permettant d'observer des états de durée de vie longue, comme les états triplets. L'invention des lasers pulsés va augmenter la résolution temporelle d'abord à la nanoseconde, puis la picoseconde pour finalement atteindre la femtoseconde, ce qui va permettre d'observer toute la dynamique interne des molécules et de comprendre des mécanismes réactionnels. En particulier Ahmed Zewail va développer la «femtochimie » permettant de détecter les états de transition et d'analyser les effets de cohérence et de contrôle de la phase de l'impulsion laser, ce qui lui vaudra le prix Nobel de chimie en 1999.

\section{Cours 2 - Photochimie moléculaire}

La photochimie est un domaine très vaste qui concerne la chimie des états excités après absorption d'un photon par la molécule. Ceci contraste avec la chimie de l'état fondamental qui se fait typiquement en chauffant les composés. Dans ce cours, seuls les grands principes sont abordés, commençant par une introduction à la structure électronique et vibrationnelle des molécules, le rôle très important de la solvatation, la 
théorie de l'état de transition, les surfaces d'énergie potentielle, les règles de Woodward-Hoffmann ainsi que la théorie des orbitales frontières de Kenichi Fukui pour expliquer les produits des réactions en fonction de la symétrie des orbitales ainsi que de leur signe. Ces principes seront illustrés par des exemples tirés de la photochimie des oléfines et alcènes. Finalement, la théorie de Marcus sur les réactions de transfert d'électron est expliquée et illustrée par des exemples expérimentaux.

\section{Cours 3 - Lumière, métal et molécules (I)}

Les plasmons de surface peuvent être compris comme de la lumière piégée à la surface d'un métal par interaction avec les électrons libres de ce dernier. Il en résulte que le champ électromagnétique du plasmon est intense à la surface du métal. Les plasmons de surface ont plusieurs avantages qui font qu'ils sont utilisés dans divers domaines allant des circuits et composants photoniques aux capteurs biomédicaux pour détecter la présence de molécules d'intérêt ou encore la détection de molécules uniques par le signal Raman exalté. En effet, les plasmons de surface permettent de concentrer l'onde électromagnétique dans un volume sub-longueur d'onde, ce qui ouvre la voie à la miniaturisation de composants optiques et l'exaltation du champ. Les propriétés des plasmons de surface peuvent littéralement être taillées sur mesure en contrôlant la structure du métal à l'échelle nanométrique. La courbe de dispersion (relation entre fréquence et vecteur d'onde) des plasmons de surface permet d'appréhender leurs propriétés et les conséquences de leurs interactions avec les molécules.

\section{Cours 4 - Lumière, métal et molécules (II)}

Les structures périodiques, comme des réseaux de trous cylindriques, dans un film métallique permettent de coupler la lumière aux plasmons de surface à des longueurs d'onde bien définies (des modes) pour un angle donné. Le spectre de transmission de réseaux de trous sub-longueurs d'onde dans des films opaques montre des pics correspondant à ces modes plasmoniques, c'est-à-dire aux longueurs d'onde où le champ électromagnétique est exalté au-dessus des trous. Il en résulte une transmission extraordinaire qui est 100 à 1000 fois plus élevée que celle attendue dans le cadre de la théorie de Hans Bethe pour des trous sub-longueur d'onde. En d'autres termes, le champ électromagnétique intense des plasmons de surface compense la faible transmission par effet tunnel des trous. Depuis la découverte de la transmission extraordinaire des trous sub-longueurs d'onde, diverses structures à base de trous ont été étudiées, allant du trou simple aux trous entourés par des sillons périodiques cylindriques (des cibles). Les cibles sont particulièrement intéressantes car elles peuvent aussi focaliser la lumière transmise dans une direction donnée définie par la courbe de dispersion. Les structures à base de trous ont trouvé diverses applications allant de photo-détecteurs ultra-rapides pour l'optoélectronique à des dispositifs capables de piéger des protéines uniques et d'observer leur changement de conformation.

\section{Cours 5 - Interactions dans les régimes de couplage faible et fort (I)}

L'électrodynamique quantique nous montre que les interactions lumière-matière forment un domaine beaucoup plus riche en possibilités que l'approche semi- 
classique ne le laisse entendre. Pour comprendre la physique en jeu, il faut rappeler les propriétés du vide selon l'électrodynamique quantique. Les interactions lumièrematière peuvent être classées dans deux régimes distincts, les régimes dits de couplage faible et fort. Dans le régime de couplage faible, les propriétés radiatives des molécules peuvent être modifiées comme l'a montré Karl Drexhage dès 1968. Il a étudié les propriétés radiatives d'un complexe d'Europium à différentes distances devant un miroir et a montré que la durée de vie et l'intensité de fluorescence varient en fonction de la densité d'états du vide. Dans le régime de couplage fort, les interactions sont si fortes qu'ils se forment des états hybrides lumière-matière, dites polaritoniques, même dans l'obscurité du champ du vide. La séparation de Rabi des états polaritoniques est traitée théoriquement par le modèle de Jaynes-Cummings. Lorsque beaucoup de molécules sont fortement couplées à un même mode d'une cavité optique, les états polaritoniques sont collectifs et la séparation de Rabi peut atteindre des énergies de l'ordre de l'électronvolt.

\section{Cours 6 - Interactions dans les régimes de couplage faible et fort (II)}

Lorsque des molécules sont fortement couplées à un résonateur optique tel qu'une cavité optique ou un mode plasmonique, et que de nouveaux états hybrides se forment, on peut s'attendre à ce que les propriétés matérielles de l'ensemble du système soient modifiées. Alors que les premières études de molécules fortement couplées datent de 1982, elles étaient focalisées sur les aspects optiques du couplage et c'est seulement depuis 2012 que le premier rapport sur la modification de propriété a été publié. Cette étude montre que la cinétique et le rendement d'une réaction photochimique est modifié sous couplage fort. Les effets du couplage fort sur la réactivité chimique est encore plus surprenant en couplant les transitions vibrationnelles des molécules dans l'infrarouge. On peut alors voir des modifications de mécanismes réactionnels et même des effets catalytiques. Les propriétés des matériaux solides peuvent aussi être changées par le couplage fort. Par exemple, la conductivité de semi-conducteurs organiques peut être exaltée ainsi que le transfert d'énergie entre molécules. Ce type de résultats ouvre de vastes perspectives pour les sciences moléculaires et les sciences des matériaux.

\section{COLLOQUE - QED-M2 «NEW QED LANDSCAPES FOR MOLECULES AND MATERIALS 》}

Le colloque "QED-M2 "New QED landscapes for molecules and materials" ", qui s'est tenu les 18 et 19 juin 2018 au Collège de France, a été centré sur les nouvelles perspectives à l'interface des sciences moléculaires, de la physique quantique et de l'électrodynamique quantique. Il a réuni des chercheurs de divers pays présentant les dernières avancées sur la thématique permettant des discussions approfondies.

- Wolfgang Wernsdorfer (KIT, Karlsruhe) : «Operating quantum states in single magnetic molecules »;

- Mario Ruben (KIT, Karlsruhe) : «Engineering molecular spin qudits »;

- Päivi Törmä (Aalto university, Helsinki) : «Bose-Einstein condensation in a plasmonic lattice »; 
- Cyriaque Genet (CNRS et université de Strasbourg) : «Chiral coupling of valley excitons with spin-momentum locked surface plasmons »;

- Francisco Garcia-Vidal (Madrid autonomous university): «Polaritons for chemistry and materials science »;

- Francis Spano (Temple university, Philadelphia): «Theory of vibronic polaritons in optical microcavities: The effects of multi-particle states »;

- Jaime Gomez-Rivas (Eindhoven institute of technology) : «Strong light-matter coupling in extended open cavities »;

- Giacomo Mazza (École polytechnique, Paris) : «Strong light-matter coupling and collective phenomena in quantum materials »;

- Xiaolan Zhong (Beihang university, Beijing) : «Non-radiative energy transfer via light-matter strong coupling »;

- Jino George (IISER, Mohali, India) : «Conductivity and photoconductivity under strong coupling »;

- Tal Schwartz (Tel Aviv university) : «Tailoring energy transport in molecules by confined photons »;

- Luis Martin-Moreno (university of Zaragoza) : «Molecular cavity quantum electrodynamics: ultrastrong coupling effects »;

- Greg Scholes (Princeton university) : «Coupling vibrational modes using light »;

- Naomi Ginsberg (UC Berkeley) : «Functional structure of energy materials through universal tracing of tortuous excitation paths »;

- Jenny Clark (Sheffield university): «Triplet pair states in organic semiconductors »;

- Vahid Sandoghdar (MPI, Erlangen) : «Photonic spook: Molecules feeling each other at large distances »;

- Guido Pupillo (université de Strasbourg et CNRS) : «Cavity enhanced transport of excitons and charge »;

- Jana Zaumseil (Heidelberg university): «Weak and strong light-matter coupling with semiconducting polymers and carbon nanotubes »;

- Claudiu Genes (MPI, Erlangen) : «Applications of collective subradiant states of coupled quantum emitters »;

- Javier Aizpurua (DIPC, San Sebastian): «Cavity-enhanced molecular optomechanics »;

- Karl Börjesson (university of Göteborg): «Selective manipulation of electronically excited states through strong light-matter interactions »;

- Atef Shalabney (Braude College, Israel): «Molecular vibrational strong coupling: novel route to modify materials properties »;

- Anoop Thomas (université de Strasbourg et CNRS) : "Chemistry under vibrational light-matter strong coupling »;

- Bill Barnes (university of Exeter) : «The vacuum as an engineering material ».

\section{PuBLications}

EBBESEN T., L'Alchimie du vide. Interactions lumière-matière en chimie physique, Paris, Collège de France/Fayard, coll. « Leçons inaugurales », 2019.

Zhong X., Chervy T., Zhang L., Thomas A., George J., Genet C., Hutchison J.A. et EBBESEN T.W., «Energy transfer between spatially separated entangled molecules », 
Angewandte Chemie International Edition, vol. 56, no 31, 2017, p. 9034-9038, DOI : 10.1002/ anie. 201703539.

Hertzog M., Rudquist P., Hutchison J.A., George J., Ebbesen T.W. et BöRJesson K., «Voltage-controlled switching of strong light-matter interactions using liquid crystals », Chemistry. A European Journal, vol. 23, no 72, 2017, p. 18166-18170, DOI : 10.1002/ chem.201705461.

Bahsoun H., Chervy T., Thomas A., Börjesson K., Hertzog M., George J., Devaux E., Genet C., Hutchison J.A. et EBbesen T.W., « Electronic light-matter strong coupling in nanofluidic Fabry-Pérot cavities », ACS Photonics, vol. 5, no 1, 2018, p. 225-232, DOI : 10.1021/acsphotonics.7b00679.

Chervy T., Thomas A., Akiki E., Vergaume R.M.A., Shalabney A., George J., DeVAuX E., Hutchison J.A., Genet C. et EBBESEN T.W., « Vibro-polaritonic IR emission in the strong coupling regime », ACS Photonics, vol. 5, no 1, 2018, p. 217-224, DOI : 10.1021/ acsphotonics.7b00677.

Chervy T., AzZini S., Lorchat E., Wang S., Gorodetski Y., Hutchison J., Berciaud S., EBBESEN T. et GENET C., « Room temperature chiral coupling of valley excitons with spinmomentum locked surface plasmons », ACS Photonics, vol. 5, n 4, 2018, p. 1281-1287, DOI : 10.1021/acsphotonics.7b01032.

Thomas A., Chervy T., AzZini S., Li M., George J., Genet C. et EbBesen T.W., « Mueller polarimetry of chiral supramolecular assembly », The Journal of Physical Chemistry C, vol. 122, no 25, 2018, p. 14205-14212, DOI : 10.1021/acs.jpcc.8b01867.

OKada D., Azzini S., Nishioka H., Ichimura A., Tsuji H., NAKamura E., Sasaki F., Genet C., EBbeSEN T.W. et YAMAMOTO Y., « $\pi$-Electronic co-crystal microcavities with selective vibronic-mode light amplification: toward Förster resonance energy transfer lasing », Nano Letters, vol. 18, no 7, 2018, p. 4396-4402, DOI : 10.1021/acs.nanolett.8b01442.

Thomas A., Lethuillier-Karl L., Nagarajan K., Vergauwe R.M.A., George J., Chervy T., Shalabney A., Devaux E., Genet C., Moran J. et Ebbesen T.W., « Tilting a ground-state reactivity landscape by vibrational strong coupling », Science, vol. 363, $\mathrm{n}^{\circ} 6427$, 2019, p. 615-619, DOI : 10.1126/science.aau7742. 
